\title{
Ein ,Freiwilliges Historisches Jahr'? Überlegungen zum historischen Lernen und Arbeiten im Rahmen gegenwärtiger Jugendfreiwilligendienste
}

\author{
Dr. Oliver Plessow
}

Universität Kassel $\mid$ FB Gesellschaftswissenschaften | Fachdidaktik Geschichte Lehrkraft für besondere Aufgaben | oliver.plessow@uni-kassel.de

Die gegenwärtige Auffächerung der Freiwilligendienste führt dazu, dass der Einsatz von Jahresfreiwilligen zunehmend auch dort möglich ist, wo der Dienst einen Schwerpunkt auf eine Beschäftigung mit der Vergangenheit legt. Der Beitrag unterbreitet einen Vorschlag, wie dieser sich abzeichnende Typus eines ,Freiwilligen Historischen Jahrs' strukturiert beschrieben werden kann. Analysiert werden geschichtskulturell geprägte Einsatzstellen und die an ihnen ausgeübten vergangenheitsbezogenen Tätigkeiten; einbezogen werden zudem die von Trägerorganisationen angebotenen begleitenden Bildungsmaßnahmen. Innerhalb der Bandbreite geschichtskulturell ausgerichteter Einsatzstellen treten Museen, Gedenkstätten und die Denkmalpflege als bedeutsame Einsatzorte hervor. Bei den Tätigkeiten stechen die Vermittlung historischer Bildung sowie die Wahrung der historischkulturellen Überlieferung heraus. Eine zentrale Rolle für den Geschichtscharakter eines Dienstes spielen zudem die Begleitprogramme. Dies gilt sogar dort, wo die Einsatzstellen selbst keinen konkreten historischen Bezug aufweisen.

Schlagwörter: Jugendfreiwilligendienste; Historisches Lernen; Geschichtskultur; FSJ Kultur; Gedenkstätten; Museen 


\section{Einführung}

Im Mai 2012 stellte Spiegel Online fünf jugendliche Freiwillige vor, die gerade ihren Dienst in einer Gedenkstätte für die Opfer des Nationalsozialismus leisteten. Zwar finden sich in der Rubrik „Schulspiegel“, die sich hauptsächlich an junge Leserinnen und Leser wendet, immer wieder einmal Artikel, welche die Freiwilligendienste einer breiteren Öffentlichkeit bekannt machen wollen. Warum gewannen aber gerade diese fünf Jugendlichen die besondere Aufmerksamkeit des Magazins? Außergewöhnlich erschien, dass Neunzehn- und Zwanzigjährige freiwillig ein Jahr ihrer Lebenszeit der Arbeit an „Orte[n] des Grauens“ widmen. Nicht ohne Bewunderung hebt der Artikel hervor, wie sich junge Menschen auch gegen Widerstände ihres Umfelds daran machen, die Vergangenheit in die Gegenwart zu holen: „Während ihre Freunde ein Studium beginnen oder um die Welt reisen, arbeiten die jungen Leute in ehemaligen Konzentrationslagern. Sie wollen verhindern, dass der Holocaust vergessen wird. Und sie wollen sich mit ihrer eigenen Geschichte auseinandersetzen“(Deuber 2012).

Selbst wenn der Dienst in einer KZ-Gedenkstätte eine sehr spezielle Form des Freiwilligenjahrs darstellt, lässt er sich in eine ganze Reihe von Freiwilligenjahrtypen einordnen, die historisches Lernen ermöglichen und befördern, darunter solche in Museen, in Archiven oder in historischen Bibliotheken. Sie alle bieten besondere Lernkontexte, die eine intensive Beschäftigung mit der Geschichte erlauben. Dass dies in diesem Umfang heute möglich ist, hängt mit der seit gut einem Jahrzehnt zu verzeichnenden Ausweitung und Diversifizierung der Diensttypen und Einsatzfelder zusammen (Stemmer 2009: 12). Diese Entwicklung hat dazu geführt, dass ein sich typischer Weise an das Ende der Schulzeit anschließendes Überbrückungsjahr immer häufiger auch von der Auseinandersetzung mit historischen Themen geprägt sein kann.

Ziel dieses Beitrags ist es, den empirisch zu fassenden Typus eines ,Freiwilligen Historischen Jahrs' strukturiert zu beschreiben und damit in der Fachöffentlichkeit zur Diskussion zu stellen. Dies ist bislang nicht versucht worden, wodurch auch weiterführende Fragen bislang kaum bearbeitet werden können - etwa zu spezifischen Formen historischer Sinnbildung im Zusammenhang dieses Lernorttyps, zum Beitrag der Dienste zur Vergesellschaftung historischer Diskurse oder auch zu ihrer Rolle als Rekrutierungsinstru- 
menten zukünftiger Multiplikatorinnen und Multiplikatoren im Bereich des historischen Lehrens und Forschens, Erinnerns und Gedenkens.

Damit ist im Kern zunächst eine genuin geschichtsdidaktische Frageperspektive eingenommen. Geschichtsdidaktisch ist die Perspektive insofern, als die Teildisziplin seit Jahrzehnten verstärkt nicht nur die Geschichtsvermittlung in der Schule erforscht, sondern darüber hinaus intensiv historischen Sinnbildungsphänomenen in der Gesellschaft als Ganzer und ihren Manifestationen nachspürt (Schönemann 2000: 35-42). Geschichtsdidaktiker fahnden heute nach Orten historischer Sinnbildungsprozesse auch außerhalb von Klassenzimmern, und zweifellos bilden Freiwilligendienste mit historischer Ausrichtung derartige Lernorte. Sie lassen sich als Elemente der Geschichtskultur betrachten, wenn man diese mit Jörn Rüsen ,als praktisch wirksame Artikulation von Geschichtsbewußtsein im Leben einer Gesellschaft" definiert (Rüsen 2008: 235).

Im Hinblick auf die Einordnung des Beitrags in die Freiwilligendienstforschung geht es hier also um das Wirkungspotenzial auf die Freiwilligen selbst (Freiwillige als „Zu Bewirkende“) - konkret: auf deren historische Sinnstiftungen - und nicht etwa um das von ihnen im Rahmen ihres Einsatzes Bewirkte (Freiwillige als „Bewirkende“, vgl. Fischer 2011: 55f.) Gefragt werden soll hier primär nach den institutionellen Möglichkeitsbedingungen, sprich: dem Rahmen, welchen bestimmte Diensttypen für historisches Lernen bieten. Welchen Baustein aus dem Komplex sozialer Systeme, die „Sinnbildung über Zeiterfahrung“ (d. h. die Ausbildung von Geschichtsbewusstsein, Rüsen 2008: 75) strukturieren, stellen historische Freiwilligenjahre dar?

Wieso ist das auch über die binnengeschichtsdidaktische Diskussion hinaus bedeutsam? Aufmerksamkeit über die Teildisziplin hinaus verdient die Frage, weil sie zu einer differenzierteren Vorstellung von der gesellschaftlichen Situierung der Freiwilligendienste beiträgt. Sie kann Praktikerinnen und Praktikern helfen, sich im Feld zu positionieren, zu anderen Akteuren in Beziehung zu setzen und überhaupt ein umfassenderes Verständnis von den Rahmenbedingungen ihres Tuns zu entwickeln. Zudem kann die Betrachtung eines ,Freiwilligenjahrs Geschichte' den Nachbardisziplinen Denkanstöße vermitteln sowie Fachdiskussionen interdisziplinär anschlussfähig halten. 
Der vorliegende Beitrag konzentriert sich dabei auf eine Auseinandersetzung mit den einjährigen Jugendfreiwilligendiensten, die immer noch den Regelfall der Dienstarten darstellen. Nicht vergessen werden darf in diesem $\mathrm{Zu}$ sammenhang, dass wir uns derzeit in einer Phase des Wandels der Freiwilligendienste befinden, die insbesondere auch einen stärkeren Zugriff des Staates (Klein 2011: 17f.) und die Ausbildung generationenübergreifender Dienste beinhaltet (Stemmer 2009: 4f.). Diese Veränderungen könnten langfristig auch die Strukturierung des Felds jener Dienste verändern, die einen Blick auf die Vergangenheit werfen. Zudem ist der Untersuchungsbereich insofern eingeschränkt, als zumindest der Einsatzort oder die Herkunft der Freiwilligen auf das Territorium der Bundesrepublik bzw. ihrer Länder beschränkt ist - dies ist nicht zuletzt den staatlichen Regelungen geschuldet, welche den gesetzlichen und organisatorischen Rahmen für die unterschiedlichen Dienste bestimmen.

In einem ersten Schritt wird es in der Folge darum gehen zu klären, ob die bestehenden Systematisierungsansätze dazu taugen, für unseren Zusammenhang einschlägige Einsichten zur Strukturierung des Felds zu befördern. Inwieweit wird bereits wahrgenommen, dass einige Dienste die Möglichkeit $\mathrm{zu}$ intensivem historischen Arbeiten und Lernen bieten, andere dagegen nicht? Erst dann kann dazu übergangen werden, selbst einen nach den Aspekten ,Arbeit' und ,Bildung' gegliederten Systematisierungsvorschlag vorzulegen. Zum Abschluss wird noch ein Schlaglicht auf die quantitative Relevanz dieser Dienste im Vergleich zur gesamten Landschaft der Freiwilligenjahrformen geworfen.

\section{Bestehende Forschungs- und Praxiskonzepte und ihre Berücksich- tigung einer historischen Perspektive}

Bevor ich also versuchen möchte, einen eigenen Einteilungsvorschlag vorzulegen, gilt es zu prüfen, inwieweit die vorhandenen Kategoriensysteme eine historische Perspektive berücksichtigen. Es liegt daher nahe, dafür zunächst den Blick auf jene Zergliederung des Felds zu richten, wie sie sich in den letzten Jahren durch das Entstehen konkurrierender Diensttypen ergeben hat. Obwohl es auch staatsferne und nicht rechtlich gerahmte Typen gibt, ist die gegenwärtige Auffächerung primär ein Ergebnis der Initiativen unterschiedlicher, bisweilen konkurrierender staatlicher Akteure. Zur in diesem Zu- 
sammenhang denkbaren Ausbildung eines spezifisch historischen Diensttyps ist es allerdings nicht gekommen.

Auszunehmen von dieser Betrachtung sind zunächst jene Typen, die thematisch nicht fokussiert sind (selbst wenn hier auch historische Projekte ihren Platz finden), also der Bundesfreiwilligendienst (BFD) als neu aufgestellter Nachfolger des Zivildienstes, der Internationale Jugendfreiwilligendienst des Bundes (IJFD) oder der Europäische Freiwilligendienst (EFD). Wo indessen eine Spezialisierung stattgefunden hat, verdankt sich dies in zwei Fällen dem Sonderinteresse einer starken staatlichen Institution: Wenn man bedenkt, wie sehr die Dienste kulturweit und weltwärts den Geschäftsbereichen des Auswärtigen Amts bzw. des Ministeriums für wirtschaftliche Zusammenarbeit und Entwicklung verpflichtet sind, wird offenbar, wie wenig ohne ein „Geschichtsministerium" für eine vergleichbare staatliche Initiative zur Einrichtung eines vergleichbaren genuin „historischen“ Diensttyps spricht.

Eine größere Chance zur Ausbildung eines separaten „Freiwilligenjahrs Geschichte" hätte beim von Bund und Ländern gemeinsam geförderten Typus der Freiwilligenjahre bestanden. Seit der gesetzlichen Neuregelung von 2002 ist im Rahmen von Freiwilligem Sozialen Jahr (FSJ) und Freiwilligem Ökologischen Jahr (FÖJ) die Ableistung eines Dienstes in gesellschaftlich relevanten Feldern möglich. Dazu zählen auch Bereiche, in denen historische Tätigkeiten situiert werden können. Explizit werden im FSJÄnderungsgesetz „Einrichtungen der Kinder- und Jugendhilfe, einschließlich der Einrichtungen für außerschulische Jugendbildung und Einrichtungen für Jugendarbeit oder [...] Einrichtungen der Gesundheitspflege und kulturelle[.] Einrichtungen (Einsatzstellen).“ genannt. ${ }^{1}$ Tatsächlich sind seit der Gesetzesänderung die Möglichkeiten der Spezialisierung aufgenommen worden und es haben sich die Sonderformen des FSJ Kultur und FSJ Politik und zuletzt auch des FSJ Schule entwickelt; in den Reihen der diesen Diensten zugeordneten Einsatzstellen kann man zurecht auch solche mit einer historischen Perspektive erwarten, wofür unten noch mehrere Beispiele folgen. Dass in diesem Zusammenhang die Ausbildung eines ,Freiwilligen Historischen Jahrs' oder eines ,FSJ Geschichte' nicht undenkbar gewesen wäre

\footnotetext{
${ }^{1}$ Vgl. hierzu.: §2 (2) FSJÄndG v. 29. Mai 2002 im BGBl. 2002 I: 1667, so auch in §3

(1) JFDG v. 16. Mai 2008 im BGBl. 2008 I: 842.
} 
(und auch weiterhin denkbar ist), zeigt die Entwicklung des einem solchen Konstrukt sicherlich zuordenbaren Freiwilligen sozialen Jahrs in der Denkmalpflege, das eine noch viel engere Diensttypdefinition aufweist und ohne Weiteres einem größeren Typ ,FSJ Geschichte' untergliedert werden könnte. $\mathrm{Zu}$ seinen Entstehungsbedingungen unten mehr.

Verlässt man den Bereich der gesetzlich fundierten bzw. gesetzliche Rahmenvorgaben aufgreifenden Einteilungen und betrachtet man, inwieweit die Trägerverbände selbst, die beaufsichtigenden staatlichen Stellen oder die bisherige Freiwilligenarbeitsforschung Kategorisierungen vorgenommen haben, so fällt auf, dass auch hier der historische Bereich nicht als eigenständige Größe wahrgenommen wird. Die Platzbörse des Bundesfreiwilligendienstes etwa kennt zwar u. a. die Sparten „Kultur“, „Soziales“, „Begegnungsstätte“ und „Jugendliche, Schule“, nicht aber „Geschichte“ oder dergleichen (BAFzA o. J.). Die Werbebroschüre des Bundesministeriums für Familie, Senioren, Frauen und Jugend (BMFSFJ) für den BFD wiederum stellt als Einsatzbereiche den „Sozialen Bereich“, den „Ökologischen Bereich“, die „Kultur“, die „Bildung“, den „Sport“ und die „Integration“ vor (BMFSFJ 2011: 24). Immerhin werden bei den genannten Beispielen im „Kultur“-Bereich (,Museum, Theaterprojekt, Kulturverein, archäologische Ausgrabung und Denkmalpflege") auch Stichworte genannt, die eine historische Ausrichtung nahe legen. Der fünfjährige Freiwilligensurvey des Familienministeriums, der allerdings bürgerschaftliches Engagement insgesamt und nicht allein Freiwilligenjahre erfasst, unterscheidet in seiner Erhebung u. a. nach den Kategorien „Kultur und Musik“, „Politik und Interessenvertretung“, „Umwelt, Naturschutz, Tierschutz“ und „Außerschulische Jugendund Bildungsarbeit“" (Gensicke/Geiss 2010: Anhang Methodenbericht 20), nicht aber nach „Geschichte und Erinnerung“ oder Ähnlichem. Grundsätzlich gilt: Wo in den einschlägigen Publikationen Themenbereiche spezifiziert werden, gibt es bislang „Geschichte“ nicht als Kategorie (z.B. Fischer 2011: 54; BKJ 2007: 16f.; Landesstiftung Baden-Württemberg 2006: 60f.). Dieser Eindruck bestätigt sich auch, wenn man den Blick weitet und auf Schemata der Jugend- und Bildungsforschung allgemein blickt (zuletzt Wischmeier/Macha 2012: 173-190).

$\mathrm{Zu}$ verzeichnen sind immerhin seit geraumer Zeit von der Geschichts- und Politikdidaktik inspirierte Anstrengungen, das Historische Lernen in der Bildungsforschung allgemein als eigenen Lerntyp zu fassen. Oft geht es 
dabei um eine Klärung des Verhältnisses zwischen historischem und politischem Lernen. Während die einschlägigen theoretischen Beiträge zunehmend das Verbindende wie das Eigenständige der jeweiligen Lerntypen reflektieren, treffen sie meist keine Aussagen über die Konkretisierungen in der Praxis oder denkbare Formate außerschulischen Engagements (Barricelli / Lücke 2011; Hellmuth/Klepp 2010: 124-136; Kühberger 2009: bes. 113115; Lange 2007).

Als Folge dieses Diskussionsstands wird ,historisch“ im Bereich der Praxis vielfach in Verbindung mit ,politisch' gedacht. Dabei findet sich die Kategorie ,historisch-politisch' oft immer noch so gebraucht, dass eher der erste Teil des Wortpaars den zweiten bestimmt, als dass ein kopulatives Verständnis vorliegt und ,historisch-politisch' als ein Subtyp der politischen Bildung erscheint (mit Verweis auf unterschiedliche Integrationsmodelle Lange 2004: 13-17, hier insb. 15f.). Für einen Blick auf die Freiwilligendienstlandschaft impliziert dies eine Verengung der Perspektive, denn allzu leicht wird hierdurch die Verbindung zwischen primär politischen und nicht primär politischen historischen Diensttypen in der Betrachtung gekappt. Einschränkend wirkt auch die der Kategorie „historisch-politisch“ eigene Konzentration auf die Phänomene der Zeitgeschichte, welche ähnliche Diensttypen mit einem Schwerpunkt in der Vormoderne oder im 19. Jahrhundert ausschließt.

\section{Möglichkeiten der Typisierung vergangenheitsbezogener Freiwilli- gendienste}

Hat demnach der Blick auf Forschungs- und Praxiseinteilungen den Anfangseindruck bestätigt, dass ein ,Freiwilligenjahr Geschichte' bislang nicht als Diensttyp (an)erkannt wird, bleibt zu fragen, an welcher Stelle eine Betrachtung, welche das Strukturierungspotenzial der Größe ,Geschichte' im Bereich des längerfristigen Freiwilligenengagements sondieren will, ansetzen kann. Einen ersten Anknüpfungspunkt bietet dafür die Verortung der Freiwilligendienste im Bereich der non-formalen bzw. halbformalen Bildung. Weder besitzen sie den Pflichtcharakter des schulischen Ausbildungssystems mit seinen Lehrplänen, staatlich examinierten Fachkräften und Abschlüssen noch den diffusen Charakter des Ad-hoc-Lernens in der Interaktion mit Familie, Peer Groups und Medien (Hafeneger 2011: 38f.; Thole 
2011: 75; Wischmeier/Macha 2012: 115-117). Bei Freiwilligendiensten ist der Verbindlichkeitscharakter höher als bei der informellen Bildung und der Bildungscharakter durch ein von den Trägern anzubietendes Begleitprogramm gewährleistet, ohne dass der Freiwilligkeitscharakter verloren ginge. Diese Definition der Freiwilligendienste, wie sie die Literatur schon seit geraumer Zeit vorgeschlagen hatte (Rauschenbach/Liebig 2002: 19f.), ist mittlerweile sogar im Jugendfreiwilligendienstgesetz niedergelegt (JFGD v. 16. Mai 2008 (BGBl. 2008 I: 842)).

Für die hier gewählte Frageperspektive ist entscheidend, dass diese besondere Aufstellung der Freiwilligendienste zur Folge hat, dass sie sich stets im Spannungsfeld von Bildung und Arbeit befinden. Daraus ergeben sich zwei Möglichkeiten: Zum einen kann zur Strukturierung des Feldes die von den Freiwilligen ausgeübte Tätigkeit daraufhin befragt werden, ob sie sich überwiegend auf die Auseinandersetzung mit der Vergangenheit richtet; und zum anderen kann eruiert werden, ob eine Auseinandersetzung mit der Vergangenheit den Kern der Bildungschance darstellt, die ein solcher Dienst bietet. Beiden Aspekten soll nachgegangen werden, denn beide können helfen, Schneisen in das Dickicht der Dienstvielfalt zu schlagen.

\subsection{Der historische Charakter der Einsatzstellen}

Die Einsatzstelle definiert den Ort, an dem der freiwillige Arbeitseinsatz erfolgt. Da die Freiwilligen im Zuge ihrer Tätigkeit informelle und nonformale Lernprozesse durchlaufen, die auf sie selbst wirken (Fischer 2011: 56), eignet sich der Charakter des Einsatzortes als Klassifizierungskategorie. Fragt man nun, welche Einsatzstellen eine dezidiert historische Ausrichtung haben und welche Subtypen hier auftreten, kann man wie gezeigt nicht einfach den existierenden Einteilungsschemata aus Forschung und Praxis folgen. Hier hilft erst eine genauere Analyse der einzelnen Einsatzstellen und der jeweiligen Tätigkeitsbeschreibungen. Grundlage der nachfolgenden Ausführungen bildet dementsprechend ein theoretisches Sampling (Glaser/Strauss 2010: 61-91) auf der Grundlage einer seit Längerem in Entstehung befindlichen, ständig erweiterten Sammlung verfügbarer Einsatzortlisten und Stellenbeschreibungen. Das untersuchte Material ist zwar zu heterogen - auch was angesichts der Spannbreite vom einfachen Listeneintrag bis zum Blog die Informationstiefe betrifft - als dass die genaue Angabe der Zahl der untersuchten Einsatzorte und Einzelstellen aussagekräftig wäre; zudem wird die Sammlung im Zuge anderer Forschungen ständig erweitert. 
Es sei jedoch aus Transparenzgründen vermerkt, dass ich zum Zeitpunkt der Abfassung insgesamt eine Auswahl von 214 Einsatzstellen, bei denen ein geschichtskultureller Bezug zu erkennen war, einer genaueren Kategorisierung unterzogen habe; eine theoretische Sättigung war nach der Sichtung von etwa 100 Einsatzstellen erreicht.

Betrachten wir als erstes jene Einsatzstellen, an denen schwerpunkthaft historisch gearbeitet oder historisches Lernen gefördert wird. Wie sind diese zu charakterisieren? Typischerweise ist das Gros der vergangenheitsbezogenen Einsatzstellen an Museen, Gedenkstätten und gelegentlich in Archiven situiert. Damit sind jene primären außerschulischen historischen Lernorte benannt, wie sie auch von der Geschichtsdidaktik regelmäßig identifiziert werden, wenn es darum geht, Geschichtslehrerinnen und -lehrer auf mögliche Partner für einen Unterricht außerhalb des Klassenraums aufmerksam zu machen (Sauer 2012: 139-149).

Im Bereich der Museen beruht eine solche Zuordnung zur Geschichtskultur zwar insofern zum Teil auf Einschätzung, als dass auch in Einrichtungen ohne kulturhistorischen Schwerpunkt eine historische Perspektive eingenommen werden kann - wie ist etwa ein FSJ Kultur im Deutschen Technikmuseum Berlin zu bewerten, das zwar eine technikgeschichtliche Ausstellung sein Eigen nennt, aber mit dem ,Technoversum' aktuell gerade einen in die Zukunft gerichteten Blick einnehmen möchte (Böndel 2010: 30f.)? Auch bei einer Platzierung in Kunstmuseen mit ihren fast immer historischen Sammlungen stellt sich diese Frage, denn nicht immer steht hier der Aspekt der Zeiterfahrung im Vordergrund. Insgesamt aber werfen viele dieser dem geschichtskulturellen Einsatzstellentyp zuzurechnenden musealen Dienstorte keine Schwierigkeiten auf: Beispiele bieten ein Einsatz in der Antikensammlung der Staatlichen Museen Berlin ebenso wie im Hamburger Museum für Kunst und Gewerbe oder im Mannheimer Reiss-Engelhorn-Museum, im Gutenberg-Museum Mainz ebenso wie im Historischen Museum Aurich oder auf dem Museumsbauernhof Wennerstorf.

Wie eingangs schon geschildert, vermag ein Einsatz in Gedenkstätten eine besondere Aufmerksamkeit auf sich zu ziehen, zumal wenn sie den Verbrechen des Nationalsozialismus gewidmet sind. Selbst wenn in Museen mehr Freiwillige eingesetzt werden als in Gedenkstätten, ist dies ein wichtiges Einsatzfeld. Kaum eine der größeren NS-Gedenkstätten möchte die Unterstützung durch Jahresfreiwillige missen, und auch viele kleinere setzen sie 
ein. (Dies ist insofern bemerkenswert, als dass Freiwillige in diesen Gedenkstätten mit oft dünner Personaldecke maßgeblich zur Aufrechterhaltung des Betriebs beitragen können.) So finden sich Dienstplätze an allen acht vom Bund geförderten Gedenkstätten innerhalb der Grenzen der heutigen BRD, an denen sich in der NS-Zeit KZ-Stammlager befunden haben (BergenBelsen, Buchenwald, Dachau, Flossenbürg, Mittelbau-Dora, Neuengamme, Ravensbrück und Sachsenhausen). Sie existieren aber auch an Orten der T4Aktion (also der sog. „Euthanasie“, hier Bernburg, Hadamar und PirnaSonnenstein), an Gedenkstätten für die frühen Lager der Jahre 1933/34 (z. B. Osthofen), an Gedenkstätten für KZ-Außen-, Zwangsarbeiter- oder Kriegsgefangenenlager (z. B. Salzgitter-Drütte, Schillstraße Braunschweig), sonstigen Terror- und Vernichtungsgedenkstätten (z. B. Gedenkstätte Deutscher Widerstand, Haus der Wannseekonferenz in Berlin) oder an eher singulären Gedenkorten wie der noch jungen Gedenkstätte am Firmensitz der KZKrematorienbauer Topf und Söhne in Erfurt. Schließlich kennen auch Gedenkstätten, welche die Geschichte der Verfolgungen in der sowjetischen Besatzungszone und der DDR bzw. die des Kalten Krieges dokumentieren, den Einsatz von Freiwilligen (z. B. Hohenschönhausen, Point-AlphaStiftung). Ein Einsatz in der Gedenkstätte Bautzen schließlich, welche die Verfolgungen beider Diktaturen dokumentiert, verbindet beide Perspektiven.

Seltener als Museen oder Gedenkstätten halten Archive Jugendfreiwilligendienstplätze vor, was zum Teil mit der im Vergleich personell schwächer ausgestatteten Archivpädagogik zusammenhängen mag (Sauer 2012: 143). Wo jede Abordnung von Lehrerinnen- und Lehrern mühsam ausgehandelt und die pädagogische Arbeit oft nebenbei geleistet werden muss, können die Kraft und das Geld fehlen, hier mehr Stellen einzurichten. Einige aber gibt es gleichwohl: Zu nennen sind etwa Stellen für ein FSJ Kultur im Hauptstaatsarchiv Stuttgart, beim sächsischen Landesbeauftragten für StasiUnterlagen, bei der DEFA-Stiftung oder im Archiv der Jugendkulturen in Berlin.

Einen besonderen Stellenwert kann im Bereich der Freiwilligendienste mit historischem Schwerpunkt das Freiwillige Soziale Jahr in der Denkmalpflege beanspruchen. Diese von den Internationalen Jugendgemeinschaftsdiensten (ijgd) und der Deutschen Stiftung Denkmalschutz gemeinsam entwickelte und bis heute getragene Dienstform ging aus einem erfolgreichen Quedlinburger Pilotprojekt aus dem Jahr 1999 hervor. Es entwickelte sich mittler- 
weile zu einem höchst erfolgreichen Modell, das zwar auch einen Schwerpunkt auf die Vermittlung in diverse Einsatzstellen in Handwerksbetrieben, bei Denkmalbehörden, Restauratoren und Museen legt, gleichzeitig aber dem gemeinsamen Lernen mehr Raum als andere Dienstformen einräumt. Die Freiwilligen sind in derzeit dreizehn Jugendbauhütten zusammengefasst - der Begriff selbst zeugt von der Anlehnung an mittelalterliche Traditionen (ijgd 2012: 32-37). Damit ist ein ausgeweitetes Bildungsprogramm in der Gruppe verbunden, das die Vermittlung kunstgeschichtlicher wie restaurierungstechnischer Kenntnisse umfasst (Nathan 2009: 58; Rossner 2006).

Den Einsatz bei Grabungen, den manche Freiwilligendienstleistende im Rahmen ihres Denkmalpflegejahrs kennenlernen, führt über zu einem Einsatzorttyp, der eine separate Betrachtung beanspruchen kann. Denn inzwischen hat auch die Archäologie Freiwilligenjahre als mögliche Form des Engagements für sich entdeckt. So helfen Freiwillige der Universität Osnabrück bei Grabungen in Kalkriese, dem mutmaßlichen Ort der Varusschlacht, und auch die Koblenzer, Speyrer und Trierer Landesarchäologie sowie die Osnabrücker Stadt- und Kreisarchäologie halten FSJ-Plätze vor. Weitere Einsatzorte finden sich an Bildungseinrichtungen, die eine dezidiert historische Ausrichtung ausmacht (z.B. das Anne Frank Zentrum in Berlin oder die Jugendgeschichtswerkstatt Spandau), bei Einrichtungen der (historischen) Kulturverwaltung (etwa bei der Generaldirektion Kulturelles Erbe Rheinland-Pfalz) oder im Bereich von Bibliotheken mit historischen Sammlungen (z.B. an der Weimarer Herzogin Anna Amalia Bibliothek oder an der Forschungsbibliothek Gotha).

Einige Sonderfälle entziehen sich einer solchen Zuordnung zu typischen geschichtskulturellen Institutionen. Ein schönes Beispiel bietet etwa das Theater ,Das letzte Kleinod' mit Sitz im niedersächsischen SchoffdorfGeestenseth, das im eigenen, Ozeanblauen Zug' Stücke auf der Basis lokaler mündlicher Überlieferungen inszeniert.

Viele der an diesen Einsatzorten abgeleisteten Jugendfreiwilligendienste sind im Bereich des seit 2002 zur Verfügung stehenden FSJ Kultur angesiedelt, dem in manchen Ländern noch das deutlich kleinere FSJ Politik zur Seite steht. Doch täuscht sich, wer meint, damit das Spektrum der Dienste, die potenziell einen geschichtskulturellen Schwerpunkt aufweisen, überblicken zu können. Zwar geben einem die über den Trägerverbund der Bundesvereinigung Kulturelle Kinder- und Jugendbildung e. V. (BKJ) erreichba- 
ren, nach Ländern geordneten Listen der Einsatzstellen im FSJ Kultur einen guten ersten Überblick über die Stellenvielfalt, doch partizipieren auch die anderen staatlich regulierten Dienste (wie im Übrigen auch einige der ungeregelten Dienstformen) am historischen Engagementbereich. Tatsächlich nutzen einige Einsatzstellen und Trägerorganisationen sogar bewusst die Vielfalt, nicht zuletzt um eine Finanzierung möglichst vieler Stellen gewährleisten zu können. An der Gedenkstätte Mittelbau-Dora in Nordhausen etwa gibt es neben einem Platz im FSJ Kultur derzeit (2012/13) zwei Plätze im Bundesfreiwilligendienst (Stiftung Gedenkstätten o. J.). In der Gedenkstätte Bergen-Belsen gab es schon 2009/10 nebeneinander ein FSJ Kultur und ein FSJ Politik (Trutz 2011: 59). Überhaupt zeichnen sich Gedenkstätten durch eine größere Varianz aus: Gerade hier finden sich häufig auch Freiwillige aus dem Ausland - vermittelt etwa im Rahmen des österreichischen Gedenkdiensts (Verein Niemals Vergessen 2012; Gedenkdienst 2012) oder durch die Aktion SühnezeichenFriedensdienste (ASF 2011: 55-60) -, wie ja auch viele deutsche Freiwillige an Gedenkstätten im Ausland tätig sind.

Bei den größeren Trägerorganisationen ist diese proaktive Nutzung der Auffächerung der Dienste in den letzten Jahren nicht zuletzt vor dem Hintergrund der Aussetzung der Wehrpflicht und der damit verbundenen Neuaufstellung von Freiwilligenprogrammen zu sehen. Profilierte Organisationen sehen sich hier eben nicht als Erfüllungsgehilfen staatlicher Programme; stattdessen geht es eher darum, für die eigenen Programme eine Förderung einzuwerben, aus welchem staatlichen Haushaltsposten diese auch kommen mag (ASF 2011: 9).

Im Ganzen ist nicht zu erkennen, dass diese in Bezug auf eine geschichtskulturelle Schwerpunktsetzung heterogene Struktur des Feldes das Ergebnis einer bewussten Entscheidung oder gar strategischen Planung ist, von Einzelinitiativen einmal abgesehen. Vielmehr handelt es sich um gewachsene Strukturen. Gleichwohl ist zu bedenken, dass für die hier aufgeführten gemeinnützigen Träger anders als die von Gesetz dazu bestimmten natürlichen Träger eine Zulassung erforderlich ist ${ }^{2}$ - hierdurch ergibt sich immerhin eine

\footnotetext{
2 Vgl. hierzu: §10 JFDG v. 16. Mai 2008 (BGBl. 2008 I: 842); §7 BFDG v. 28. April 2011 (BGBl. I: 687)
} 
Plessow, Ein ,Freiwilliges Historisches Jahr‘?

gewisse Steuerung, wenn auch keine, die einem geschichtskulturellen Horizont Rechnung trüge.

\subsection{Der historische Charakter der einzelnen Tätigkeitsbereiche}

Der Blick auf die institutionelle Aufstellung der Einsatzstellen leitet über zu einer Analyse der jeweiligen Tätigkeitsbereiche. Erst diese kann darüber Aufschluss geben, inwieweit eine Stelle wirklich einen „Reichtum an Erfahrungs-, Lern- und Bildungsmöglichkeiten“ aufweist (Stemmer 2009: 8). Mit welchen Tätigkeiten, die einen Bezug zur Vergangenheit aufweisen, werden die jugendlichen Freiwilligendienstleistenden in der Praxis betraut? Die Sichtung von Stellenbeschreibungen (die in der Folge nicht einzeln nachgewiesen werden) kann hier wichtige Hinweise geben; ihnen zur Seite gestellt werden können Erfahrungsberichte und Blogs, die im Rückblick festhalten, worin das Engagement jeweils bestand. Die Analyse zeigt dabei, dass schon die einzelne Stelle in der Regel recht unterschiedliche Tätigkeiten umfasst. Dies ist nicht zuletzt der Orientierungsfunktion der Jugendfreiwilligenjahre geschuldet, da die Freiwilligen ja vielfältige Einblicke in die Berufspraxis erhalten sollen. Gleichzeitig zeitigt die Gesamtschau wiederkehrende Muster: Gerade in der Vielfalt offenbaren sich immer wieder dieselben Grundtypen vergangenheitsbezogener Tätigkeiten. Deshalb kann die folgende Systematisierung helfen, das jeweilige Tätigkeitsfeld von Freiwilligen besser zu fassen.

Zum Ersten zu nennen ist die Tätigkeit als Mittler historischen Lernens. Viele Dienstorte treten bereits institutionell als solche auf und bieten dementsprechend Freiwilligen die Möglichkeit, sich aktiv in ihre pädagogischen Programme einzubringen. Bei Gedenkstätten, die ihre Freiwilligen im Bereich ihrer pädagogischen Angebote einsetzen, oder in einschlägigen Jugendbildungsstätten wie der Internationalen Jugendbegegnungsstätte in Oświęcim/Auschwitz oder dem Max-Mannheimer-Studienzentrum in Dachau wird die Tätigkeit die eines Mittlers historisch-politischen Lernens sein (und damit wie gesagt den Fokus fast zwangsläufig auf die Zeitgeschichte legen); möglich ist aber auch eine weniger epochenspezifische Ausrichtung. In Museen etwa werden viele Freiwillige im Bereich von Besucherführungen und der Museumspädagogik eingesetzt, wobei die thematische Ausrichtung sich natürlich nach der des jeweiligen Museums richtet; im Schwäbischen Volkskundemuseum Oberschönenfeld oder im niedersächsischen Freiluftmuseum Kiekeberg zum Beispiel ist das dann eben keine historisch- 
politische, sondern eine der Kultur- und Alltagsgeschichte. Insgesamt kommt dem Tätigkeitsfeld der Vermittlung auch dort besondere Bedeutung zu, wo der Dienstort als Ganzes einen anderen Arbeitsschwerpunkt hat. Denn die weitaus meisten Freiwilligen an geschichtskulturellen Einsatzstellen sind mit irgendeiner Form von Vermittlung betraut. Im weiteren Sinne zählt auch die Präsentation historischer Inhalte, etwa in Form von Ausstellungen, zu dieser Vermittlungsfunktion.

Einen zweiten wichtigen Typ bilden jene Tätigkeiten, welche mit der Wahrung der historisch-kulturellen Überlieferung verbunden sind; im Englischen wird dieser Bereich oft mit dem Begriff Heritage beschrieben. Die institutionelle Spezialisierung hat es mit sich gebracht, dass wir für die Wahrung, Erschließung und Dokumentation der schriftlichen und bildlichen Hinterlassenschaft sowie der mobilen und der immobilen materiellen Hinterlassenschaft typischerweise separate Facheinrichtungen entwickelt haben. Hier ist als erstes an Museen, Archive und Denkmalschutzbehörden zu denken. Eine Partizipation von Freiwilligen an Heritage-Aufgaben liegt vor, wenn es eben nicht (nur) um die (Mit-)Konzeption und Durchführung der jeweiligen pädagogischen Programme geht, sondern (auch) um eine Übernahme von Teilen der Kernaufgaben der jeweiligen Einrichtungen, also des Ergrabens, Aufbewahrens, Restaurierens, Archivierens, Sammelns, Beschreibens, Inventarisierens usw. In einigen Fällen gehören solcherlei Tätigkeiten auch dort zum Aufgabenspektrum, wo man sie zuerst vielleicht nicht vermuten würde. So wünscht sich die Gedenkstätte Buchenwald etwa von Freiwilligen eine Mithilfe bei der fachkundigen Pflege der denkmalgeschützten Bausubstanz. Auch Fälle, in denen ein anderer Bewahrungsaspekt die Arbeit dominiert, als vom Format des Dienstjahrs her ersichtlich, treten auf. So nutzte eine Freiwillige der Jugendbauhütte Mühlhausen einen Großteil ihrer Arbeitszeit nicht etwa für Restaurierungsarbeiten, sondern für Archivrecherchen im Zuge einer Dokumentation von Kirchenglocken und Standbildern (Nathan 2009: 58f.).

Dieser Fall zeigt bereits, dass manchmal zum Dritten Freiwilligen die Möglichkeit gegeben wird, auch ohne einschlägige Berufsqualifikationen Erfahrungen in historischer Forschung zu sammeln. So bietet ihnen das Goetheund Schiller-Archiv Weimar an, an einem der laufenden wissenschaftlichen Editionsprojekte mitzuwirken, während die Gedenkstätte KZ Osthofen eigenständige themenspezifische Archivrecherchen ermöglicht. Viele Ge- 
denkstätten begreifen sich sowohl als Lern- als auch als Forschungsorte, und Freiwillige sollen beide Aspekte selbst erproben.

Bereits ins Nicht-Historische reichen zum Vierten jene Tätigkeiten im historischen Kulturmanagement, die nur ausnahmsweise im Mittelpunkt des Aufgabenportfolios stehen, aber an fast allen Einsatzstellen zumindest einen gewissen Teil von ihm darstellen. Zu denken ist hier an Dinge wie die Übernahme der Korrespondenz oder die Pflege der EDV, an Ausstellungsorganisation, Öffentlichkeitsarbeit und dergleichen Organisations- und Verwaltungsaufgaben mehr.

Wenn sie mehrere Plätze anbieten, differenzieren manchmal bereits die Einsatzstellen selbst die Aufgabengebiete so aus, dass unterschiedliche Zuschnitte mit unterschiedlichen Schwerpunkten entstehen. Hierzu ein Beispiel: Die Stiftung Haus der Geschichte der Bundesrepublik Deutschland bietet am Standort Bonn jährlich vier Plätze an, die neben den nicht spezifisch historischen Tätigkeiten „Veranstaltungskoordination“ und „IT“ historisch-vermittelnde Aufgaben im Bereich der „Museumspädagogik“ sowie historisch-bewahrende bzw. aufarbeitende Aufgaben im Bereich „Objektdokumentation“ und „Werkstätten“ umfassen; am Standort Berlin hingegen kommt mit der Tätigkeitsbeschreibung „Dokumentation“ nur ein Aspekt zum Tragen.

Die doppelte Kategorisierung des Aspekts des historischen Arbeitens im Zuge von Jugendfreiwilligendiensten nach dem Institutionscharakter der Einsatzstelle einerseits und dem Typ der Tätigkeitsfelder andererseits ist ein probater Weg, die Erscheinungsformen der gegenwärtigen Praxis zu beschreiben und weitere Forschungen zu eröffnen. Verknüpfen ließe sich dies in zukünftigen Studien mit weiteren Kategorisierungen, wie sie die Geschichtswissenschaft bzw. die Geschichtsdidaktik entwickelt haben. Ein weiteres Zuordnungsraster böten beispielsweise die Dimensionen historischer Wahrnehmung (Pandel 2009: 47f.), also Herrschaft, Wirtschaft, Alltag usw. Denkbar wäre weiterhin eine Zuordnung nach epochenbezogenen inhaltlichen Schwerpunktbereichen. Mittelfristig reizvoll wäre es dann, den hier gewählten Ansatz mit diesen beiden Kategorien zu verknüpfen, um etwa nachvollziehen zu können, ob die Beschäftigung mit dem mittelalterlichen Rittertum, mit der frühneuzeitlichen bäuerlichen Gesellschaft, mit der Herrschaftspraxis der DDR oder eben mit der Zeit des Nationalsozialismus bereits durch die ausgeübten Tätigkeitstypen bestimmte Zugriffsweisen - und 
damit auch die Chance zu einer bestimmten Einflussnahme auf das Geschichtsbild - standardisiert. Zumindest für die Auseinandersetzung mit der NS-Zeit mit ihrer ausgeprägten Gedenkstättenlandschaft ist dies sicherlich schnell plausibel zu machen.

\subsection{Die pädagogische Begleitung durch die Träger}

Wenn also Freiwilligendienste jungen Menschen an der Schwelle zum Erwachsenwerden aufgrund der Chance einer „Bewährung durch Tun“ bedeutsame „Bildungs- und Orientierungsangebote“ machen (Stemmer 2009: 7) nicht zuletzt dafür werden sie staatlich gefördert -, dann ist es erkenntnisfördernd, die jeweilige Schwerpunktsetzung im Bereich der Bildung ebenfalls als Kategorie zur Einschätzung der Bedeutsamkeit einer historischen Perspektive heranzuziehen. Daher tritt ein Blick auf die von den Trägern verantworteten organisierten Bildungsmaßnahmen neben einen Blick auf die Ausgestaltung der Arbeitsplätze, denen allerdings zweifellos ebenfalls ein Bildungsaspekt zu Eigen ist (Stemmer 2009: 7f.). Dies ist durchaus durch die Praxis gerechtfertigt: Für die staatlich legitimierten Freiwilligendienste ist die Verpflichtung, der Arbeit im Freiwilligenjahr ein Bildungsprogramm an die Seite zu stellen, inzwischen einheitlich gesetzlich geregelt; für den BFD ist sogar eine Mindestzahl von 25 Seminartagen vorgegeben ${ }^{3}$. Der Umstand, dass die Verantwortung für die Bildungsarbeit nicht bei der Einsatzstelle, sondern bei der Trägerorganisation liegt, ist bedeutsam, weil dies andere Lernformen - nämlich in der Gruppe - und eigene Schwerpunktsetzungen ermöglicht. Bei Trägerorganisationen, die mit einem recht hohen eigenen Bildungsanspruch aufwarten (zur Typisierung der Trägerorganisationen Wischmeier/Macha 2012: 176), kann dies eine ganz eigene und eben auch historische Prägung des Dienstjahres zur Folge haben.

In Bezug auf die Auseinandersetzung mit Geschichte bedeutet dies nun nicht, dass jegliches historische Versatzstück in der Bildungsarbeit als Anhaltspunkt für die Existenz eines ,Freiwilligen Historischen Jahrs' gewertet werden müsste. Denn der gar nicht einmal so seltene Fall, dass im Zuge eines pädagogischen Begleitprogramms eine Gedenkstätte oder ein historisches Museum aufgesucht wird (siehe z.B. die regelmäßigen Besuche von

\footnotetext{
${ }^{3}$ Vgl. hierzu: §3 (2)/§4 (2) JFDG v. 16. Mai 2008 (BGBl. 2008 I: 842); §4 BFDG v.
} 28. April 2011 (BGBl. I: 687) 
Gruppen des Internationalen Bundes in der Gedenkstätte Breitenau bei Kassel, Hanke/Richter 2012: 6), ist zwar geschichtsdidaktisch nicht minder interessant, aber an dieser Stelle systematisch nicht relevant. Betrachtet werden müssen vielmehr jene Fälle, bei denen das Beiprogramm überwiegend auf Zeiterfahrung fokussiert ist oder in denen die Gesamterfahrung als historisches Bildungserlebnis definiert wird.

Festzuhalten ist zunächst, dass nicht alle Trägerorganisationen, die Einsatzstellen mit geschichtskulturellem Bezug betreuen, ein historisches Beiprogramm anbieten. So sieht die pädagogische Rahmenkonzeption der Bundesvereinigung Kulturelle Kinder- und Jugendbildung, des Trägerverbunds für das FSJ Kultur, zwar vor, dass die Freiwilligen auch dazu angehalten werden, gesellschaftliche Fragen wie die Rolle bürgerschaftlichen Engagements oder die Partizipation durch Bildung zu reflektieren. Dass eine solche $\mathrm{Zu}$ kunftsperspektive auch historisch fundiert werden kann, gerät aber nur peripher in den Blick (BKJ 2011: 3, 5). Anders sieht es dort aus, wo eine Trägerorganisation als Vermittlerin zwischen Freiwilligen und institutionellem Träger des Dienstorts tritt, die eine dezidiert historische Perspektive einnimmt. Hier kann es geschehen, dass die Erfahrungen im Dienst als historische Sinnbildungsangebote konzeptualisiert werden. Zwei Paradebeispiele, die auch angesichts des Umfangs ihrer Aktivitäten besonders herausstechen, seien hier genannt: Zum Ersten betrifft dies die Jugendbauhütten der ijgd mit ihren bereits erwähnten kultur-, bau- und kunsthistorischen Seminaren; allein schon augrund des größeren zeitlichen Umfangs kann hier das Beiprogramm erhöhtes Gewicht beanspruchen. Noch eindrücklicher ist dies bei der Aktion Sühnezeichen-Friedensdienste, die ihr Versöhnungskonzept bereits im Namen trägt und am Ausgang der 1950er Jahre zu den Wegbereitern der Ausbildung von Auslandsdiensten gehörte (zur Entwicklung eingehend jetzt bei Legerer 2011). Diese erfolgten und erfolgen bis heute unter dem Leitsatz, jenen durch konkretes Handeln Gutes zu tun, die durch den Zweiten Weltkrieg und den staatlichen Terror der Nationalsozialisten zu Opfern wurden, „Der Verein ist eine Organisation, die - in Aufnahme und Weiterführung des Gründungsaufrufes von 1958 - die Versöhnung mit den vom nationalsozialistischen Deutschland überfallenen wie von der Vernichtung bedrohten Völkern und Menschengruppen und die Entwicklung der Friedensfähigkeit zum Ziel hat," wie es das Vereinsziel bestimmt (ASF 2009: 1). Das bedeutet aber gar nicht einmal, dass der Einsatz im geschichtskulturellen Feld erfolgen muss. Zwar vermittelt ASF zahlreiche Freiwillige an nati- 
onale und internationale NS-Gedenkstätten, die Kernidee ist aber immer noch, einen direkten Dienst am Menschen zu leisten, was sich durch die Betreuung von Überlebenden und durch Arbeitseinsätze in Altersheimen, mit Behinderten, in sozialen Projekten mit Benachteiligten und dergleichen ausdrückt. Insofern aber alle gemeinsam eine Auswahl und ein anspruchsvolles vergangenheitsbezogenes Seminarprogramm durchlaufen, sind auch hier vorzügliche Rahmenbedingungen für intensives historisches Lernen gegeben.

\section{Zum Stellenwert historischer Freiwilligendienste im Gesamtspekt- rum der Diensttypen}

Skizzenhaft soll nun zum Abschluss versucht werden, eine vorsichtige Aussage darüber zu treffen, welcher Stellenwert solchen Diensten, die eine Auseinandersetzung mit der Vergangenheit prägt, im Gesamtspektrum der Dienste zukommt. Dass ein solcher Dienst in der Lage ist, mediale Aufmerksamkeit auf sich zu ziehen, hat der Beginn dieses Beitrags gezeigt. Auch die große mediale Präsenz der Arbeit von Aktion SühnezeichenFriedensdienste ist zu vermerken. Ein quantitatives Schlaglicht auf die Zahl der betroffenen Einsatzstellen fällt angesichts der Fragmentierung der Freiwilligendienstarten, der sich gerade in diesen Jahren der Umstrukturierung ständig wandelnden Zahlen, der mangelnden Vollständigkeit verfügbarer Gesamtlisten von Einsatzstellen und der manchmal schwierigen Einschätzung des Ausmaßes, in dem eine Einsatzstelle geschichtskulturell geprägt ist, schwer. Da jährlich mehrere Zehntausende junge Menschen die Möglichkeit wahrnehmen, einen langfristigen Dienst anzutreten (Stemmer 2009: 43 - also noch vor Einführung des BFD -; Hieronimus 2012: 1), mögen alle etwaigen ,Freiwilligen Historischen Jahre' wie ein Randphänomen erscheinen. Wenn man aber bedenkt, dass ganze Diensttypen wie der EFD oder das FÖJ jährlich nur Teilnehmerzahlen im hohen Hunderter- oder niedrigen Tausenderbereich aufweisen, lohnt es zumindest, ein paar Überlegungen anzustellen.

Von den Schwierigkeiten, alleine vom FSJ Kultur auf die Gesamtsituation zurückzuschließen, ist bereits gesprochen worden. Dennoch geben die von einigen BKJ-Landesvereinigungen vorgehaltenen Einsatzstellenlisten wichtige Anhaltspunkte: Betrachtet man etwa die Übersicht der Landesvereini- 
gung Kulturelle Jugendbildung Niedersachsen e.V. (LKJ Niedersachsen 2011), zeigt sich, dass sich von den dort aufgeführten 137 niedersächsischen Einsatzstellen im FSJ Kultur (120 Plätze werden hier jährlich gefördert) sechzehn bis neunzehn einen Schwerpunkt auf das Historische legen - je nachdem, welche Museen man in die Zählung aufnimmt. Neun (bzw. zwölf) dieser Einsatzstellen sind in Museen verortet, drei in Gedenkstätten, zwei im Bereich der Archäologie, eine im Bereich Archivwesen/Dokumentation und eine in dem besagten zeitgeschichtlichen Theaterprojekt. In Rheinland-Pfalz sind von den 2013/14 vorgesehenen 90 Einsatzstellen immerhin 23 historisch geprägt, darunter 14 in Museen, drei in der Archäologie, drei im Kulturmanagement sowie je eine in einer Bildungsstätte, in einem Archiv und in einer Gedenkstätte (Kulturbüro o. J.). In Berlin kann man von 79 Stellen in der Saison 2011/12 16 geschichtskulturellen Einsatzstellen zuordnen, darunter zehn in Museen, drei in Bildungsstätten, zwei in Archiven und eine in einer Gedenkstätte (LKJ Berlin o. J.). Insgesamt gibt es bundesweit im Jahr ca. 1500 FSJ-Kultur-Plätze (BKJ 2011a: 9), von denen sich schätzungsweise 250 bis 300 einer historischen Schwerpunktsetzung zuordnen lassen, und zwar zu einem großen Teil in Museen. Hier gilt es allerdings zu bedenken, dass das FSJ in der Denkmalpflege hier nicht eingerechnet ist und Freiwilligendienste in Gedenkstätten häufig nicht über das FSJ Kultur laufen und so nur unzureichend in die Zählung eingehen. Denn auf diese beiden Typen entfallen sicherlich mit den Museen zusammen die weitaus meisten Teilnehmenden. Hält man nun den FSJ-Kultur-Zahlen die Zahlen der beiden großen Träger entgegen, die einen klaren Geschichtsbezug aufweisen, komplettiert dies das Bild: In den Jugendbauhütten der ijgd versammelten sich 2011/12 311 Freiwillige (ijgd 2012: 59). ASF schickte 179 Freiwillige in die Saison 2011/12 und empfing in Deutschland 29 internationale Freiwillige (ASF 2012: 10). Insgesamt durchlaufen sicher über 700 Teilnehmende gegenwärtig jährlich das empirische Konstrukt eines ,Freiwilligen Historischen Jahrs' und haben in dieser Zeit ausgedehnte Möglichkeiten zu historischer Sinnbildung.

\section{Synopse und Ausblick}

Diese Freiwilligenzahl rechtfertigt es, von einem Einsatzfeld zu sprechen. Das Schaubild (Abb. 1) visualisiert synoptisch den Systematisierungsvorschlag, der geeignet ist die Binnengliederung dieses Felds aufzuzeigen. Die 
Betrachtung der Aspekte ,Arbeit' und ,Bildung' hat gezeigt, welche einzelnen Engagementbereiche hier von hervorgehobener Bedeutung sind und welche Tätigkeiten diesen Typ von Freiwilligenjahr kennzeichnen.

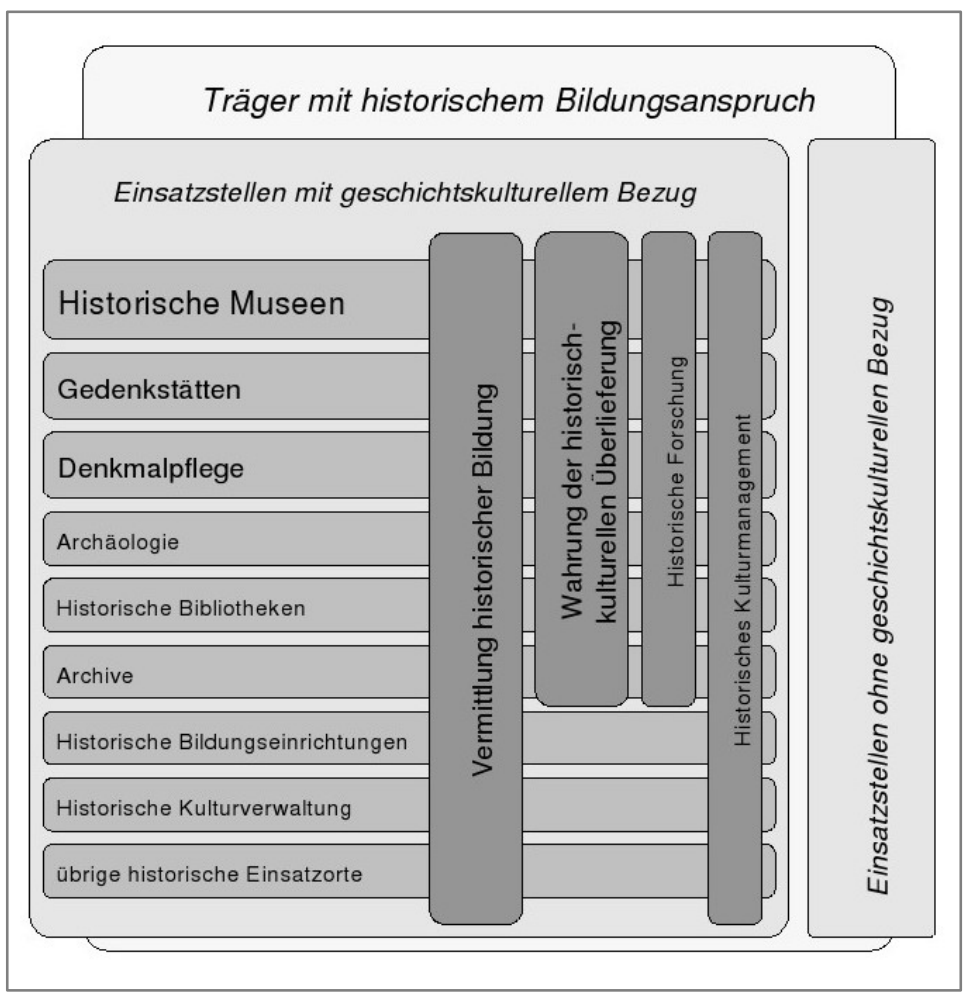

Abb. 1: Vorschlag einer Systematisierung der vergangenheitsbezogenen Einsatzstellen im Jugendfreiwilligendienst. Quelle: Eigene Darstellung

Indes ist damit erst der Grund für weitere Forschungen gelegt. Unklar ist vorerst, inwieweit die Einsatztypen unterschiedliche historische Sinnbildungsmuster generieren. Gerne würde man wissen, ob sich hier Jugendliche sammeln, die schon über ein stark differenziertes Geschichtsbewusstsein verfügen und hier Entfaltungsmöglichkeiten finden. Doch auf die Frage, inwieweit die Freiwilligen den historischen Aspekt zum Auswahlkriterium ihrer Stellenwahl machen, lässt sich bislang keine einheitliche Antwort 
geben. Immerhin geben die vergangenheitsbezogenen Rekrutierungsmechanismen der profilierten Trägerorganisationen hier einen Hinweis: Wer sich etwa bei Aktion Sühnezeichen-Friedensdienste bewirbt, muss sich schon vorher ausgiebig mit der Bedeutung insbesondere der Shoah für die eigene historischen Sinnbildung auseinandergesetzt haben, sonst würde er oder sie das Auswahlverfahren nicht überstehen. An anderer Stelle weisen Selbstaussagen einiger Freiwilliger darauf hin, dass der Freiwilligendienst - vielleicht im FSJ Kultur noch eher als in anderen Dienstformen? - tatsächlich noch eine Orientierungsfunktion in der persönlichen Berufs- und Selbstfindung einnimmt (Fritzsch 2010: 51). Vielfach wird aber auch deutlich, dass ein einschlägiges Freiwilligenjahr den ersten Schritt zum Studium eines historischen Faches darstellt (Nathan 2009: 60).

Überhaupt sollte man in Zukunft verstärkt erforschen, inwieweit Freiwilligenjahre einen wichtigen Fokalisationspunkt für spätere Multiplikatorinnen und Multiplikatoren historischer Bildung bilden. Nur verwiesen sei hier auf die Schlüsselrolle, welche sich Aktion Sühnezeichen Friedensdienste für die pädagogische Arbeit in Gedenkstätten erarbeitet hat. Dies kann hier nicht vertieft werden, allerdings sei nur abschließend darauf hingewiesen, dass ein beträchtlicher Teil des hauptamtlichen pädagogischen Personals insbesondere der größeren NS-Gedenkstätten sich aus ASF-Ehemaligen zusammensetzt. ${ }^{4}$ Dies böte berufsbiographischen Fragestellungen genauso wie der Ideengeschichts- und Netzwerkforschung noch so manchen interessanten Ansatzpunkt.

\section{Literaturverzeichnis}

Aktion Sühnezeichen - Friedensdienste (2009): Satzung des Vereins, Berlin.

Aktion Sühnezeichen - Friedensdienste (2011): Jahresbericht 2011, Berlin.

Bundesamt für Familie und zivilgesellschaftliche Aufgaben (о. J.): Platzbörse, http://www.bundesfreiwilligendienst.de/platzboerse.html (Zugriff am 24. Nov. 2012)

${ }^{4}$ Darunter finden sich die gedenkstättenpädagogische Diskussion und Literatur stark prägende Personen wie Daniel Gaede von der Gedenkstätte Buchenwald, Elke Gryglewski von der Gedenk- und Bildungsstätte Haus der Wannseekonferenz oder Thomas Lutz, Leiter des Gedenkstättenreferats der Stiftung Topographie des Terrors. 
Voluntaris, Jg. 1, 1/2013, Aufsätze

Bundesvereinigung Kulturelle Kinder- und Jugendbildung (2007): Engagement plus Tatkraft. Empirische Ergebnisse aus dem Engagementfeld Kultur, Remscheid/Berlin.

Bundesvereinigung Kulturelle Kinder- und Jugendbildung (2011): Pädagogische Rahmenkonzeption des FSJ Kultur, Remscheid.

Bundesministerium für Familie, Senioren, Frauenund Jugend (2011): Zeit, das Richtige zu tun. Freiwillig engagiert idn Deutschland - Bundesfreiwilligendienst. Freiwilliges Soziales Jahr. Freiwilliges Ökologisches Jahr, Berlin.

Barricelli, Michele und Martin Lücke (2011): Historisch-politische Bildung, in: Handbuch Außerschulische Jugendbildung, hrsg. v. Benno Hafeneger, Schwalbach/Ts., S. 325-343.

Böndel, Dirk (2010): Technoversum - Das Museum der Zukunft, in: Museums Journal, 24. Jg., Heft 1, S. 30-32.

Deuber, Lea (2012): „Das Kapitel Rassismus ist noch nicht abgeschlossen “ - Freiwilligendienst in KZ-Gedenkstätte, in: Spiegel Online, 31.05.2012, http://www.spiegel.de/schulspiegel/leben /freiwilliges-soziales-jahr-in-kz-gedenkstaette-a-834498.html (Zugriff am 18. Nov. 2012).

Fischer, Jörn (2011): Freiwilligendienste und ihre Wirkung - vom Nutzen des Engagements, in: Aus Politik und Zeitgeschichte 48, S. 54-62.

Fritzsch, Tobias (2010) Schule, Studium, Arbeit ...doch dann kam alles anders, in: Erinnern! Aufgabe, Chance, Herausforderung Rundbrief der landeseigenen Gedenkstätten in SachsenAnhalt, Heft 1, S. 51-52.

Gedenkdienst (2012): Einsatzstellen, http://www.gedenkdienst.at/index.php?id=17 (Zugriff am 26. Nov. 2012).

Gensicke, Thomas und Sabine Geiss (2010): Hauptbericht des Freiwilligensurveys 2009. Ergebnisse der repräsentativen Trenderhebung zu Ehrenamt, Freiwilligenarbeit und Bürgerschaftlichem Engagement, München.

Glaser, Barney G. und Anselm L. Strauss (2010): Grounded Theory. Strategien qualitativer Forschung, 3. Aufl., Bern.

Hafeneger, Benno (2011): Lernen, Bildung und Jugend, in: Handbuch Außerschulische Jugendbildung. Grundlagen - Handlungsfelder - Akteure, hrsg. v. dems., Schwalbach/Ts., S. $29-42$.

Hanke, Annika und Gunnar Richter (2012): Bericht aus der Arbeit der Gedenkstätte Breitenau im Jahre 2011, in: Verein zur Förderung der Gedenkstätte und des Archivs Breitenau e.V. Rundbrief 31, S. 5-33.

Hellmuth, Thomas und Cornelia Klepp (2010): Politische Bildung. Geschichte, Modelle, Praxisbeispiele, Wien-Köln-Weimar.

Hieronimus, Dana (2012): Woher? - Wohin?: Ein Blitzlicht auf die Freiwilligendienste Kultur und Bildung, in: BBE-Newsletter, Heft 8, http://www.b-b-e.de/fileadmin/inhalte/aktuelles/2012 /04/n108_hieronimus.pdf (Zugriff am 20. Nov. 2012).

Internationale Jugendgemeinschaftsdienste (2012): Jahresbericht 2011 - Neue Perspektiven entdecken, Berlin. 
Plessow, Ein ,Freiwilliges Historisches Jahr“?

Klein, Ansgar (2011): Veränderungen in den Jugendfreiwilligendiensten. Zum Verhältnis von Staat und Zivilgesellschaft am Beispiel der Freiwilligendienste, in: Jugendfreiwilligendienste. Herausforderungen und Positionen, hrsg. v. Uwe Slüter, Düsseldorf, S. 13-18.

Kühberger, Christoph (2009): Kompetenzorientiertes historisches und politisches Lernen. Methodische und didaktische Annäherungen für Geschichte, Sozialkunde und Politische Bildung, 2. Aufl., Innsbruck-Wien-Bozen.

Kulturbüro Rheinland-Pfalz der Landesarbeitsgemeinschaft (LAG) Soziokultur und Kulturpädagogik (о. J.): Teilnehmende Einrichtungen in Rheinland-Pfalz, www.fsjkulturrlp.de/einsatzstellen (Zugriff am 19. Nov. 2011)

Landesstiftung Baden-Württemberg (2006): „Erfahrungen, die's nicht zu kaufen gibt!“ Bildungspotenziale im freiwilligen Engagement junger Menschen. Fachtagung 16. und 17. Juni 2005 in der Evangelischen Akademie in Bad Boll, Stuttgart.

Landesvereinigung Kulturelle Jugendbildung Berlin (o. J.): Aktuelle Einsatzstellen, http://www.lkj-berlin.de/fsjkultur/einsatzstellenliste/ (Zugriff am 19. Nov. 2011).

Landesvereinigung Kulturelle Jugendbildung in Niedersachsen (2011): Übersicht der Einsatzstellen in Norddeutschland, http://lkjnds.de/index.php?fsj_kultur_einsatzstellen (Zugriff am 18. Nov. 2012).

Lange, Dirk (2004): Historisch-politische Didaktik. Zur Begründung historisch-politischen Lernens, Schwalbach/Ts. 2004.

Lange, Dirk (2007): Historisches Lernen, in: Inhaltsfelder der Politischen Bildung, hrsg. v. Volker Reinhardt, Baltmannsweiler, S. 103-110.

Legerer, Anton (2011): Tatort: Versöhnung. Aktion Sühnezeichen in der BRD und in der DDR und Gedenkdienst in Österreich, Leipzig.

Nathan, Carola (2009): Botschafter für den Denkmalschutz. Die Jugendbauhütten feiern ihren zehnten Geburtstag, in: Monumente, Heft 5/6, S. 58-60 (nochmals erschienen in Monumente Online. Magazin der Deutschen Stiftung Denkmalschutz, Ausg. Juni, http://www.monumente-online.de/09/03/streiflichter/jugendbauhuetten_deutsche_stiftung _ denkmalschutz.php (Zugriff am 25. Nov. 2012)).

Pandel, Hans-Jürgen (2009): Dimensionen der Wahrnehmung, in: Wörterbuch Geschichtsdidaktik, hrsg. von Ulrich Mayer u. a., 2. Aufl., Schwalbach/Ts., S. 47f.

Rauschenbach, Thomas und Reinhard Liebig (2002): Freiwilligendienste - Wege in die Zukunft? Gutachten zur Lage und Zukunft der Freiwilligendienste für den Arbeitskreis Bürgergesellschaft und Aktivierender Staat der Friedrich-Ebert-Stiftung, Bonn.

Rossner, (2006): Jugend baut. Das Freiwillige Jahr in der Denkmalpflege, in: Monumente Online. Magazin der Deutschen Stiftung Denkmalschutz, Ausg. März, http://www.monumenteonline.de/06/02/02_jugend_baut.php.

Rüsen, Jörn (2008): Was ist Geschichtskultur? Überlegungen zu einer neuen Art, über Geschichte nachzudenken, in: Historische Orientierung, 2. Aufl., Schwalbach/Ts, S. 233-258. 
Voluntaris, Jg. 1, 1/2013, Aufsätze

Sauer, Michael (2012): Geschichte unterrichten: Eine Einführung in die Didaktik und Methodik, 10. Aufl., Seelze-Velber.

Schönemann, Bernd (2000): Geschichtsdidaktik und Geschichtskultur, in: Geschichtskultur. Theorie - Empirie - Pragmatik, hrsg. v. Bernd Mütter, Bernd Schönemann und Uwe Uffelmann, Weinheim, S. 26-58.

Stemmer, Philipp (2009): Freiwilligendienste in Deutschland. Eine Expertise zur aktuellen Landschaft der Inlands- und Auslandsfreiwilligendienste in Deutschland, Freiburg.

Stiftung Gedenkstätten Buchenwald und Mittelbau Dora, Gedenkstätte Mittelbau-Dora (o. J.): Praktikum - FSJ Kultur - BFD, http://www.buchenwald.de/871/ (Zugriff am 26. Nov. 2012).

Thole, Werner (2011): Bildung - theoretische und konzeptionelle Überlegungen. Zur Pädagogik der Ermöglichung von Mündigkeit und Anerkennung, in: Handbuch Außerschulische Jugendbildung. Grundlagen - Handlungsfelder - Akteure, hrsg. v. Benno Hafeneger, Schwalbach/Ts., S. 67-86.

Trutz, Tobias (2011): Ein Freiwilliges Soziales Jahr in der Gedenkstätte Bergen-Belsen, in: Stiftung Niedersächsische Gedenkstätten. Jahresbericht 2010, Celle, S. 59.

Verein Niemals Vergessen (2012): Einsatzstellen Deutschland, http://www.gedenkdienst.com/? cat=15 (Zugriff am 26. Nov. 2012).

Wischmeier, Inka und Hildegard Macha (2012): Außerschulische Jugendbildung. Eine Einführung. München. 
Plessow, Ein ,Freiwilliges Historisches Jahr“?

\begin{abstract}
Oliver Plessow

A Volunteer Year in History? Systematic Considerations on Learning and Working in the Field of History within the Framework of Present-day Youth Voluntary Services
\end{abstract}

Today's diversification of youth voluntary services in Germany has made youth volunteering increasingly possible in those domains where the service puts a specific emphasis on dealing with the past. The article proposes a model by which the formation of this evolving field of historical youth services can be described. An analysis of the placements in the realm of history culture shows that these are notably to be found in museums, memorial sites and historic conservation agencies. Typical activities comprise the mediation of history and the preservation of the historico-cultural tradition. In determining the historical character of a service, the supporting educational programs also play a pivotal role. This may be true even where the placements themselves carry no tangible historical character.

Keywords: Youth volunteering; historical learning; history culture; FSJ Kultur (voluntary service year in culture); memorial sites; museums 